\title{
A self-consistent transport model for molecular conduction based on extended Huckel theory with full three-dimensional electrostatics
}

Zahid, F.; Paulsson, Magnus; Polizzi, E.; Ghosh, A.W.; Siddiqui, L.; Datta, S.

Published in:

Journal of Chemical Physics

Link to article, DOI:

$10.1063 / 1.1961289$

Publication date:

2005

Document Version

Publisher's PDF, also known as Version of record

Link back to DTU Orbit

Citation (APA):

Zahid, F., Paulsson, M., Polizzi, E., Ghosh, A. W., Siddiqui, L., \& Datta, S. (2005). A self-consistent transport model for molecular conduction based on extended Huckel theory with full three-dimensional electrostatics. Journal of Chemical Physics, 123(6), 064707. https://doi.org/10.1063/1.1961289

\section{General rights}

Copyright and moral rights for the publications made accessible in the public portal are retained by the authors and/or other copyright owners and it is a condition of accessing publications that users recognise and abide by the legal requirements associated with these rights.

- Users may download and print one copy of any publication from the public portal for the purpose of private study or research.

- You may not further distribute the material or use it for any profit-making activity or commercial gain

- You may freely distribute the URL identifying the publication in the public portal 


\title{
A self-consistent transport model for molecular conduction based on extended Hückel theory with full three-dimensional electrostatics
}

\author{
F. Zahid $^{\text {a) }}$ \\ School of Electrical and Computer Engineering, Purdue University, West Lafayette, Indiana 47907 \\ M. Paulsson \\ Department of Micro and Nanotechnology, Technical University of Denmark, DK-800 Lyngby, Denmark
}

E. Polizzi, A. W. Ghosh, L. Siddiqui, and S. Datta

School of Electrical and Computer Engineering, Purdue University, West Lafayette, Indiana 47907

(Received 19 January 2005; accepted 26 May 2005; published online 16 August 2005)

\begin{abstract}
We present a transport model for molecular conduction involving an extended Hückel theoretical treatment of the molecular chemistry combined with a nonequilibrium Green's function treatment of quantum transport. The self-consistent potential is approximated by CNDO (complete neglect of differential overlap) method and the electrostatic effects of metallic leads (bias and image charges) are included through a three-dimensional finite element method. This allows us to capture spatial details of the electrostatic potential profile, including effects of charging, screening, and complicated electrode configurations employing only a single adjustable parameter to locate the Fermi energy. As this model is based on semiempirical methods it is computationally inexpensive and flexible compared to ab initio models, yet at the same time it is able to capture salient qualitative features as well as several relevant quantitative details of transport. We apply our model to investigate recent experimental data on alkane dithiol molecules obtained in a nanopore setup. We also present a comparison study of single molecule transistors and identify electronic properties that control their performance. (C) 2005 American Institute of Physics. [DOI: 10.1063/1.1961289]
\end{abstract}

\section{INTRODUCTION}

The emerging field of molecular electronics has generated both fundamental and applied interests in recent years. A wide range of molecular current-voltage $(I-V)$ characteristics from ohmic conduction in quantum point contacts, ${ }^{1}$ semiconducting $I$ - $V$ in conjugated aromatic thiols ${ }^{2}$ (including quantized conductance in single molecules ${ }^{3}$ ), to insulating behavior in alkane thiol chains ${ }^{4}$ have been reported. Some interesting device characteristics such as rectification, ${ }^{5}$ switching ${ }^{6}$ negative differential resistance on a silicon substrate, ${ }^{7}$ and even transistor action on three terminal systems $^{8-10}$ have also been demonstrated. In addition, exciting physics has been observed with molecules showing the Kondo effect, ${ }^{11,12}$ spin valve behavior, ${ }^{13}$ vibronic effects, ${ }^{14-16}$ and sensing applications. ${ }^{17}$ Many different experimental approaches have been developed over the years such as break junctions, ${ }^{2,18-20}$ scanning probes, ${ }^{21-23}$ nanopores, ${ }^{6}$ and a host of other methods. It is important to develop practical simulations tools in parallel to address molecular conduction both qualitatively for insights and quantitatively for predictability.

A number of theoretical models have been developed for calculating the $I-V$ characteristics of molecular wires using semiempirical $^{22,24-27}$ as well as first-principles theory. ${ }^{28-34}$ There are advantages and disadvantages of using either $a b$ initio or semiempirical models. Ab initio models are based on first-principles electronic structure calculations and can provide quantitatively accurate descriptions of molecular

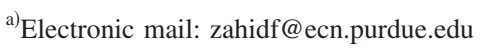

conduction without any fitting parameter, in the mean-field regime. There are disadvantages, however, namely, (i) most of these models are computationally expensive, and thus calculations are time consuming; (ii) it is difficult to describe both the molecular device and the contact electrodes at the same footing using first-principle theory, although there have been efforts in this direction; ${ }^{29,31,33}$ and (iii) the $a b$ initio models are typically not flexible to readily handle uncertainties and imperfections in the measurement geometry or electronic structure, or to incorporate complicated three terminal device geometries. On the other hand, the major advantages of semiempirical models are that these are simple, flexible, and computationally inexpensive. These models can provide a reasonable description of molecular conduction employing only a few fitting parameters. It is imperative, however, that these parameters be kept to a minimum, that they are transferable between different chemical environments and between bulk and surfaces, and that the models incorporate the full three-dimensional (3-D) self-consistent electrostatics. Subject to these attributes, semiempirical models can also become useful tools in understanding molecular conduction quantitatively. We believe that there is merit to pursuing both $a b$ initio and semiempirical approaches at this stage.

We have previously explored both ab initio ${ }^{29,35}$ and semiempirical $^{22,36-38}$ treatments of molecular conduction. Our first approach in the semiempirical line was to develop a non-self-consistent model (termed as Huckel $I-V 1.0)^{22}$ based on extended Hückel theory (EHT) where the potential inside the molecule is assumed to be flat and the voltage division is described with a constant number $\eta$. Although self- 
consistency was not explicitly considered, it was in effect subsumed into the parameter $\eta$. Later we modified this model by including an explicit self-consistent charging term in the potential profile, with $\eta$ now denoting just the Laplace contribution (Huckel $I-V$ 2.0) ${ }^{36}$ However, the potential profile was still assumed to be flat across the molecule. Both of these models are available for public use through Purdue Nanohub at www.nanohub.org. We have now further improved on the model by modifying the self-consistent potential description to include the full 3-D electrostatics leading to nontrivial spatial variations in the potential profile. This model is termed as Huckel $I-V 3.0$ which is a logical progression of our earlier models. Huckel $I-V 3.0$ now includes a proper treatment of the full 3-D electrostatic potential profile inside the molecule, and can include the influence of third (gate) terminals. In addition, it provides the advantages of simplicity, computational inexpensiveness, and ease of use as one expects from semiempirical codes. Almost all codes of Huckel I-V 3.0 are simple MATLAB codes. ${ }^{39}$ No special system requirements are needed to run these codes. A complete transport calculations for an average-sized molecule on any computer system with standard memory and speed should not take more than several hours.

In this paper we present our Huckel $I-V 3.0$ model and showcase its strengths and features. The formalism and the calculational procedures of the model are explained in Sec. II. In Sec. III we apply our model to quantitatively explain the $I-V$ characteristics of alkane dithiol molecules measured in a nanopore setup. ${ }^{14,40}$ It is worth mentioning that in one of our previous works ${ }^{37}$ we applied Huckel I-V 3.0 model for the quantitative study of another set of experimental data (asymmetric $I-V$ observed in a break junction experiment). ${ }^{19}$ In Sec. IV we present calculations on single molecule transistors comparing the performance of two molecules of similar length but of very different electronic structures. Though no direct connection is made with any experimental results on transistors, we believe it serves two useful purposes: (i) it demonstrates our ability to do three terminal calculations with proper electrostatics; and (ii) it explains the basic physics of a molecular transistor in a quantitative approach. Finally, we summarize our results in Sec. V.

\section{FORMALISM AND MODEL}

In this section we present a comprehensive description of Huckel $I-V 3.0$ model, its basic formalism and procedures. This model is based on a combination of EHT method ${ }^{41}$ and NEGF formalism along with a self-consistent description of the electrostatics. The nonequilibrium Green's function (NEGF) formalism accounts for quantum transport in molecular conductors out of equilibrium whereas EHT accounts for the electronic structures of the molecule and the contacts. We start by describing the basic structure of a molecular conductor (see Fig. 1). The molecule is assumed to be connected to contact electrodes (source and drain) through chemical bonding whereas the gate electrodes around it are electrically insulated from the molecule by oxide materials. The whole system can be thought of as a hollow 3-D box with electrode plates as its sides inside which the molecule is

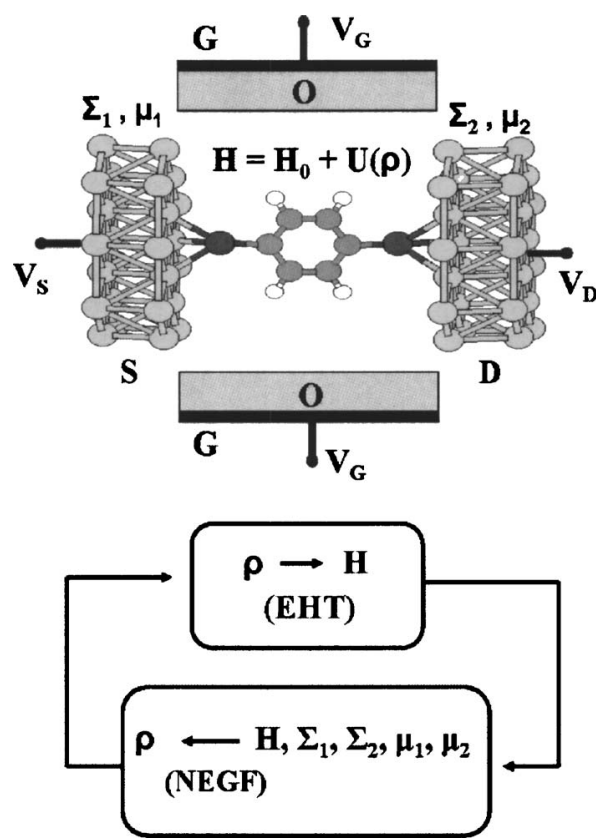

FIG. 1. The basic scheme of our self-consistent calculations along with a basic structure of a molecular conductor. The molecule is connected between two gold contacts with gate electrodes around it. The schematic shows the partitioning of the system into two parts: device and contacts. The device part is represented by the molecular Hamiltonian $H_{0}$ and the selfconsistent potential $U(\rho)$ whereas the self-energy terms $\Sigma_{1,2}$ describe the effects of contacts on the device. The self-consistent calculations are performed with a combination of NEGF and EHT in a two-step process.

placed. The sides of this box then define the boundary of the system. The dimension of the box is set by the molecular length, width, and the oxide thickness. Typically, for a two terminal system (i.e., when the gate electrodes are far away), the dimension of the box is set to be $100 \AA \times 100 \AA \times L$ where $L$ is the molecular length.

Our formalism allows us to partition the system into two separate and independent parts: a suitably defined "device" part (the molecule) and the "contacts" (the electrodes) ${ }^{29}$ The device is described by a Hamiltonian matrix $H$ and a overlap matrix $S$ obtained from any suitable quantum chemistry method, either $a b$ initio or semiempirical. The self-energy functions $\Sigma_{1,2}$ are used to describe the effect of contacts on the device with corresponding broadening functions defined as $\Gamma_{1,2}=i\left(\Sigma_{1,2}-\Sigma_{1,2}^{\dagger}\right)$. In Huckel $I-V$ 3.0, both device and contacts are described by the EHT method. The self-energy terms are calculated for semi-infinite $\mathrm{Au}$ (111) contacts using a recursive technique. ${ }^{36}$ The device Hamiltonian can be further divided into two parts: the core Hamiltonian $H_{0}$ and the self-consistent potential $U_{\mathrm{SC}}=U(\rho)$ which is a functional of the density matrix $\rho$. The full self-consistent procedure is comprised of two steps (see Fig. 1). Step 1 is to calculate the self-consistent potential given a density matrix using a suitable scheme (presented below) whereas in step 2, the density matrix is obtained using the NEGF formalism ${ }^{42}$ from the Green's function $G$ with contact electrochemical potentials $\mu_{1,2}$ and Fermi functions $f_{1,2}$. A contour integration technique $^{43}$ is employed to perform the energy integration for the calculation of the density matrix. ${ }^{44}$ From the converged Green's function all the important transport properties such as density of states, transmission, and current are cal- 
culated using the standard NEGF equations for coherent transport as given below:

$$
\begin{aligned}
& G(E)=\left(E S-H-\Sigma_{1}-\Sigma_{2}\right)^{-1} \\
& \rho=(1 / 2 \pi) \int_{-\infty}^{\infty} d E\left(f_{1} G \Gamma_{1} G^{\dagger}+f_{2} G \Gamma_{2} G^{\dagger}\right) \\
& f_{1,2}(E)=\left[1+\exp \left(\left(E-\mu_{1,2}\right) / k_{B} T\right)\right]^{-1} .
\end{aligned}
$$

The number of electrons $N$ and the steady-state current $I$ are then given by

$$
\begin{aligned}
& N=2(\text { for spin }) \times \operatorname{trace}(\rho \mathrm{S}) \\
& I=\frac{2 e}{h} \int_{-\infty}^{\infty} d E T(E, V)\left[f_{1}(E)-f_{2}(E)\right],
\end{aligned}
$$

where the transmission $T$ is given by

$$
T(E, V)=\operatorname{trace}\left(\Gamma_{1} G \Gamma_{2} G^{\dagger}\right) .
$$

\section{A. Self-consistent potential}

When an external bias is applied to a molecular conductor two kinds of effects take into place, (1) charging effect: the total number of electrons in the system starts to vary due to the flow of electrons from or to the contacts and this in turn shifts the molecular energy levels by an amount depending on the charging energy of the system; and (2) screening effects: the molecule gets polarized under the influence of the applied bias even if the total number of electrons remains unchanged. The magnitude and the direction of the polarization depend on the magnitude and polarity of the applied field. The self-consistent potential of the molecule should take both of these effects into account since the transport properties can depend sensitively on the potential inside the molecule. $^{45}$

The self-consistent potential in Huckel $I-V 3.0$ is divided into three parts as follows:

$$
U(\Delta \rho)=U_{\text {Laplace }}+U_{\text {Poisson }}(\Delta \rho)+U_{\text {Image }}(\Delta \rho),
$$

where $\Delta \rho$ represents the change in density matrix under bias, $\rho-\rho_{\text {eq }}$. As the self-consistent potential is a functional of $\Delta \rho$, it describes only the change in the potential under bias and at equilibrium it remains zero. The first term in Eq. (4) represents a standard Laplace potential and it satisfies the following equation:

$$
\nabla^{2} U_{\text {Laplace }}=0
$$

$$
\text { with } \begin{aligned}
U_{\text {Laplace }} & =-q V_{S} \quad \text { at the source } \\
& =-q V_{D} \quad \text { at the drain } \\
& =-q V_{G} \quad \text { at the gate. }
\end{aligned}
$$

The last two terms in Eq. (4) are parts of a standard Poisson potential. Here, we have divided it up in two separate parts to facilitate our calculation procedures. The Poisson term, for the moment [see the description of the CNDO (complete neglect of differential overlap) method below], is assumed to be well described by point charges in free space as follows:

$$
U_{\text {Poisson }}(\vec{r})=\sum_{\bar{r}^{\prime}} \frac{q^{2} \Delta \rho\left(\bar{r}^{\prime}\right)}{4 \pi \epsilon_{0}\left(\bar{r}-\bar{r}^{\prime}\right)} .
$$

The last term in Eq. (4) represents the image correction due to the presence of metal electrodes around the molecular structure and it is calculated by solving the following equation:

$$
\nabla^{2} U_{\text {Image }}=0
$$

with $U_{\text {Image }}=-U_{\text {Poisson }}(\vec{r})$ at the electrodes. This way of partitioning guarantees that the net potential in Eq. (4) obtained by adding Eqs. (5)-(7) has the correct boundary conditions.

Before getting into the detailed description of each term in Eq. (4) we would like to point out that the main difference between Huckel $I-V 3.0$ and our earlier models (Huckel $I-V$ 1.0 and 2.0) lies in the description of the self-consistent potential. In Huckel I-V 1.0 (Ref. 22) the self-consistent potential $U_{\mathrm{SC}}$ is given by

$$
U_{\mathrm{SC}}=\eta V_{\mathrm{appl}},
$$

where $\eta$ is a constant with any value from 0 to 1 and the potential inside the molecule is assumed to be flat. Charging and screening effects are not directly included in the description except in an averaged sense through the parameter $\eta$. Equivalently, the parameter $\eta$ can be incorporated into the contact electrochemical potentials $\mu_{1,2}$ instead, which is what was actually done in Huckel $I-V$ 1.0. In Huckel $I-V 2.0$ (Ref. 36) the expression for $U_{\mathrm{SC}}$ is modified to explicitly include the charging effects as follows:

$$
U_{\mathrm{SC}}=U_{0}\left(N-N_{\mathrm{eq}}\right)+\eta V_{\mathrm{appl}},
$$

where $U_{0}$ is the single electron charging energy of the system. The potential profile inside the molecule is still assumed to be flat. Gate electrodes were not considered in either of the two models.

Comparisons of the potential profile and the energy levels for a test system [gold-phenyl dithiol (PDT)-gold] in all of our three semiempirical models are presented in Figs. 2 and 3. These two figures clearly demonstrate that the description of electrostatics in Huckel $I-V 3.0$ is more accurate and complete compared to our earlier Huckel $I$ - $V$ models. From Fig. 2 we observe that the spatial features of the potential are present in Huckel $I-V 3.0$ whereas in the other two models, the potential profile remains flat inside the molecule. In Fig. 3 , the change in energy levels under applied bias [specifically, the change in the energy level right below the highest occupied molecular orbital (HOMO) for this particular case] shows the presence of screening effects in Huckel $I-V$ 3.0.

The Laplace and image potentials are obtained by solving Eqs. (5) and (7), respectively, with appropriate boundary conditions. These equations are solved using the Poisson module of the 3-D finite element simulator NESSIE. ${ }^{47}$ The 3-D real-space mesh has been adapted to account for the oxide layers, treated in a continuum approximation. Also, the atomic sites of the molecule are treated as point charges in free space. After finite element discretization, the resulting linear systems are solved using the preconditioned conjugate gradient method with incomplete Cholesky preconditioner. The advantage of using the finite element method (FEM) for 


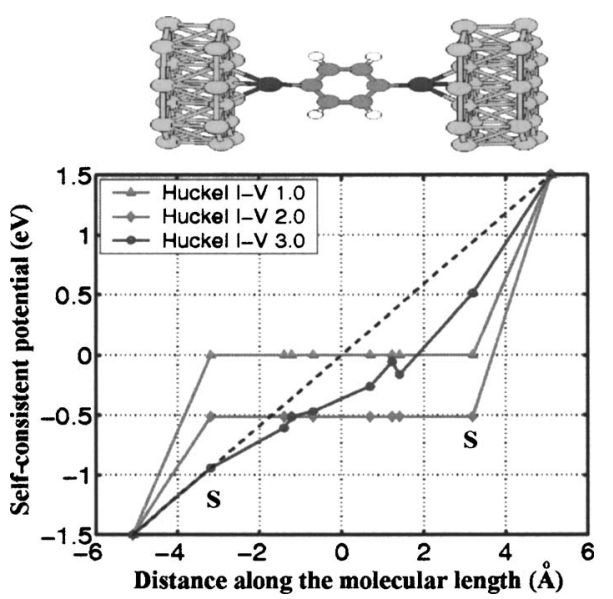

FIG. 2. A comparison of the potential profiles for the gold-PDT-gold system (shown at the top) in our EHT-based models under the applied bias of $+3 \mathrm{~V}$. The dotted line shows the applied (Laplace) potential. The " $S$ " notation indicates the position of the two sulphur end groups. The bias polarity is defined as positive when the applied voltage on the left contact is positive. Same convention of bias polarity is maintained throughout this paper. The spatial features of the potential profile are captured in Huckel $I-V 3.0$ whereas in our earlier models, the potential profile is assumed to be flat inside the molecule. Note that the device region only contains the PDT molecule. $^{46}$

the Laplace and image potentials is that it is computationally inexpensive and thus allows a large number of grid points without slowing down the calculation process. Furthermore, it can be applied to any complicated structure such as three terminal geometries with relative ease. It should be pointed out here that the inclusion of image potential in our model does not make any significant differences. We estimate that the charging energy gets lowered by around $0.1 \mathrm{eV}$ due to the image effects.

In order to solve Eqs. (5) and (7) in real space we need to transform the density matrix and the self-consistent potential from orbital space to real space and vice versa. This transformation procedure is approximated as follows:

$$
\sum_{\mu}(\rho S)_{\mu \mu}^{M}=\rho\left(r_{M}\right)
$$



FIG. 3. A comparison of energy levels as a function of applied bias for the gold-PDT-gold system in our EHT-based models. Solid line, Huckel $I-V$ 3.0; dotted line, Huckel $I-V$ 2.0. In Huckel $I-V 1.0$ the energy levels remain constant under applied bias (not shown). The electrochemical potentials for the left and right contacts are shown by $\mu_{1}$ and $\mu_{2}$. Due to the inclusion of spatial features in Huckel $I-V 3.0$ the energy levels move in a complicated but more accurate manner under applied bias whereas in Huckel I-V 2.0, all the energy levels move in the same direction by a constant value.

$$
\begin{aligned}
& U_{\mu \mu}^{M}=U\left(r_{M}\right) \\
& U_{\mu \nu}=\int \phi_{\mu}(\bar{r}) U(\bar{r}) \phi_{\nu}(\bar{r}) \mathrm{d} \bar{r} \cong S_{\mu \nu} \frac{\left(U_{\mu \mu}+U_{\nu \nu}\right)}{2} \\
& \phi_{\mu}, \phi_{\nu}=\text { atomic orbitals } \\
& r_{M}=\text { position of the atomic site } M .
\end{aligned}
$$

Because of the inherent problems with infinite potential at point charges, Eq. (6) is substituted by the CNDO (complete neglect of differential overlap) method, ${ }^{48,49}$ of which only the Hartree potential (i.e., the Coulomb interaction) is being utilized in our treatment. Both the charging and the screening effects are incorporated into this term. In CNDO approximation, this Poisson term becomes:

$$
\begin{aligned}
& U_{\text {Poisson }}^{M}(\Delta \rho)=(\Delta \rho)_{M} \gamma_{M}+\sum_{A}(\Delta \rho)_{A} \gamma_{M A} \\
& \text { with }(\Delta \rho)_{M}=\sum_{\mu}(\Delta \rho)_{\mu \mu}^{M} \\
& \gamma_{M}=\left(\mu_{M}^{2} \mid \mu_{M}^{2}\right) \\
& \gamma_{M A}=\left(\mu_{M}^{2} \mid \mu_{A}^{2}\right) \\
& M, A=\text { atomic sites } \\
& \mu=\text { Slater-type atomic orbital. }
\end{aligned}
$$

The notations used in Eq. (11) are consistent with Ref. 49. The two electron integrals $\gamma$ 's are the CNDO parameters which are obtained from the experimental data and empirical fitting. Given the distance between two atomic sites $M$ and $A$ and their CNDO parameters $\gamma_{M}$ and $\gamma_{A}$, the two-center two electron integrals can be calculated by using MatagaNishimoto approximation: ${ }^{49}$

$$
\gamma_{M A}=\frac{q^{2}}{4 \pi \epsilon_{0} R_{M A}+\frac{2 q^{2}}{\gamma_{M}+\gamma_{A}}},
$$

which describes the smearing of the charge over the atomic orbitals at short distances and approaches the Coulomb limit for point charges [Eq. (6)] at large distances. The CNDO approximation allows us to capture the main physical characteristics of the Coulomb interaction without requiring computationally expensive two electron integrals. It also avoids the need to solve 3-D Poisson's equation in real space which can also be computationally very expensive because of the sharp features of the Slater-type atomic orbitals. The CNDO treatment describes both the $\pi$ and $\sigma$ valence electrons and thus can be added to the EHT molecular Hamiltonian seamlessly.

The position of the Fermi energy relative to the molecular levels needs to be obtained self-consistently even at equilibrium. Whenever a molecule is connected to the contacts some amount of charge transfer takes place which in turn initiates an energy band lineup. As a result of this band lineup the system attains equilibrium with a single Fermi 
level $E_{f}$ throughout. The precise location of the equilibrium Fermi level depends very sensitively on many factors such as surface conditions of the contacts, nature of bonding between the molecule and the contacts, geometrical fluctuations of the structure, and environmental conditions. In the absence of detailed information of all these factors in most experiments on molecular conduction, it is not only justifiable but in fact necessary to treat the position of $E_{f}$ as an adjustable parameter. Hence, in Huckel $I-V$ 3.0, we use a fitting parameter $V_{c}$ that allows us to move the molecular energy levels up or down rigidly relative to the contact Fermi level. This is the only fitting parameter used in our model which is added to the molecular Hamiltonian (only on the molecular part and not on any part of the contacts) as a constant potential at equilibrium.

\section{I-V CHARACTERISTICS OF ALKANE DITHIOL MOLECULES}

A thorough and detailed experimental study of the $I-V$ characteristics of alkane-based molecules (both monothiol and dithiol) in a nonopore setup has been reported recently. ${ }^{14,40} \mathrm{An}$ inelastic electron-tunneling spectroscopic (IETS) study ${ }^{14}$ has also been performed on alkane dithiol molecules which shows the presence of molecules inside the pore sandwiched between two metallic electrodes. We study some of these experimental results, focusing mainly on the dithiol data, using our Huckel $I-V 3.0$ model. The reasons for focusing only on the dithiol molecules (specifically, octane dithiol) are twofold. The presence of the thiol group on both ends of the molecule helps to form chemisorbed bonding with gold contacts on both sides. This gives us a well-defined bonded structure that is easy to simulate theoretically, as opposed to unstable physisorbed contacts that do not correspond to a definite geometry-optimized structure. Secondly, the above-mentioned IETS studies are reported only on the dithiol molecules at this time and this makes the experimental results for the dithiol molecule much more reliable.

Although IETS can confirm the presence of dithiol molecules inside the nanopore in between two metallic contacts, there still remain many other issues to be experimentally sorted out, such as the exact number of molecules inside the pore (assumed to be in the order of several thousands), the nature of their bonding with the electrodes on both sides, and the surface conditions of the electrodes. In order to simulate the nanopore results we assume, for want of sufficient knowledge, that all the octane dithiol molecules inside the pore form strong and ideal chemisorbed bonds with gold contacts on both sides. We further assume that the molecules are far enough apart that each of them conducts current independently. Accordingly, we introduce a new fitting parameter, the effective number of perfectly bonded molecules inside the pore. Each end sulphur atom is assumed to sit over the middle of a gold triangle lying in an ideal, single-crystal (111) surface with the gold-sulphur bond length set to the optimized value of $2.53 \AA .^{50}$ It is worth noting that the binding energies of sulphur to other sites, such as on top of a gold atom or on the bridge between two gold atoms, are not significantly higher and could well be thermodynamically ac-

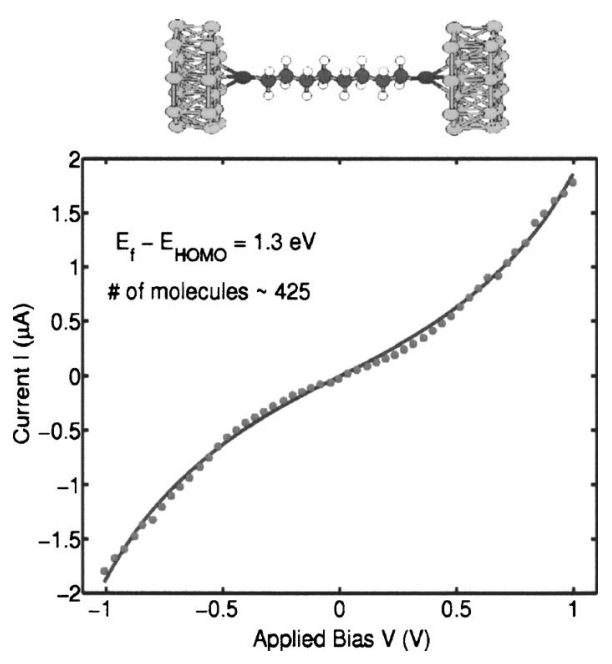

FIG. 4. I- $V$ characteristics for the gold-ODT-gold system (shown at the top). Solid line: theoretical calculations using our Huckel $I-V 3.0$ model; dots: experimentally obtained data in a nanopore setup. Two fitting parameters have been used for this match. One is $V_{c}$ which shifts the molecular energy levels and sets $E_{f}-E_{\mathrm{HOMO}}$ to $1.3 \mathrm{eV}$. The other one is the effective number of molecules $(\sim 425)$ by which the current is multiplied to obtain the total current inside the nanopore. It is assumed that each molecule in the pore is strongly connected to the gold contacts on both sides and they are conducting independently of each other. In reality, one could have a lot more molecules poorly connected and conducting much less current individually.

cessible at room temperature. There is little experimental work on characterizing the gold surfaces in molecular conduction experiments. We adopt a convenient geometry suggested by energy minimization, with the caveat that the results are quite sensitive to details of the bonding geometry.

Experimental data of the ionization potential (IP) and electron affinity (EA) are not available for the octane dithiol (ODT) molecule. Hence, we compare our results of initial energy band lineup (before molecule-gold bonding and charge transfer) with the results from a density functional theory (DFT). We use GAUSSIAN (Ref. 51) for the DFT calculations employing a Becke-Perdew-Wang (BPW) functional with a $6-31 \mathrm{G}^{*}$ basis. The HOMO-LUMO (lowest unoccupied molecular orbital) gap of the ODT molecule in EHT is $10.77 \mathrm{eV}$ whereas in DFT, it is $7.23 \mathrm{eV}$. In EHT, the $E_{f}-E_{\text {Номо }}$ value comes out to be $3.05 \mathrm{eV}$ with the gold Fermi energy calculated to be $-9.5 \mathrm{eV} .{ }^{36}$ On the other hand, in DFT, the $E_{f}-E_{\text {Номо }}$ value is given by $1.12 \mathrm{eV}$ with the gold Fermi energy set to its experimental value of $-5.1 \mathrm{eV}$. For a general discussion of the energy-level comparison between EHT and ab initio methods, please see Ref. 36.

Our $I-V$ calculations for the octane dithiol (ODT) molecule show excellent agreement with the experimental results, as is evident from Fig. 4. In order to get the best fitting of the $I-V$ the molecular levels are shifted by $V_{c}=1.55 \mathrm{eV}$, which in turn sets $E_{f}-E_{\mathrm{HOMO}}=1.3 \mathrm{eV}$. In addition, the current conducted by a single molecule is multiplied by a factor of $\sim 425$, a fitting parameter corresponding to the effective number of well-contacted molecules, to get the total current inside the pore. The adjustable parameter $V_{c}$ is reasonable, given that even for well-studied systems such as silicon transistors, it is common to adopt a threshold voltage adjustment in the industry to account for the Fermi level position uncertainties. It is, however, difficult to justify the value for the 

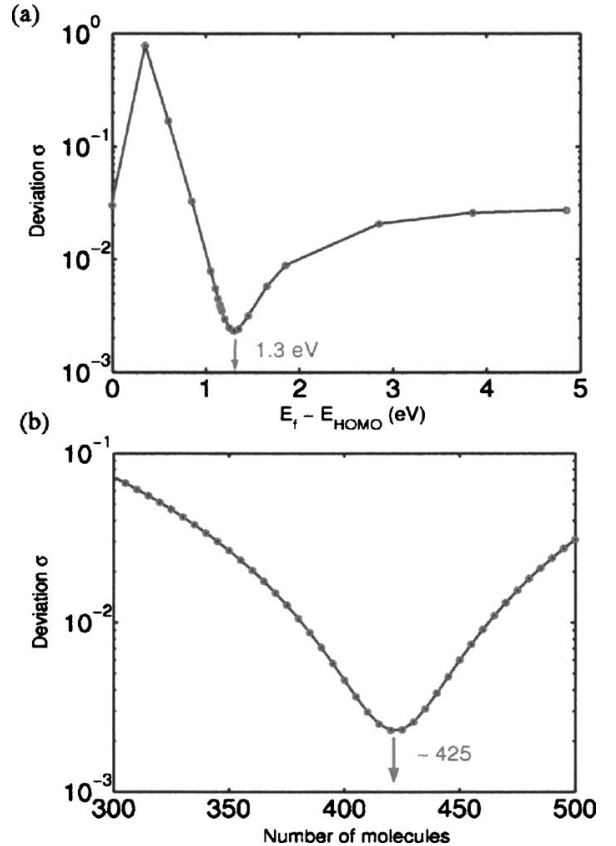

FIG. 5. A close look of the fitting: (a) the deviation $\sigma$ as a function of $E_{f}$ $-E_{\mathrm{HOMO}}$ showing a clear minimum at $E_{f}-E_{\mathrm{HOMO}}=1.3 \mathrm{eV}$; (b) the deviation $\sigma$ as a function of the effective number of molecules for the particular value of $E_{f}-E_{\text {Номо }}=1.3 \mathrm{eV}$ showing a best fit for around 425 well-contacted molecules. Deviation is defined as the sum of the square of the differences between the theoretical and experimental current values at every bias.

effective number of molecules due to lack of adequate information of the details of the pore. From the experimental value of the diameter of the pores the number of molecules inside the pore can be estimated to be of the order of several thousands. However, it is unlikely that all of these molecules are well connected at both ends to the contacts. Besides, we observe in another recent experimental study ${ }^{52}$ that the magnitude of the current through a single octane dithiol molecule is around $40 \mathrm{nA}$ at $1 \mathrm{~V}$, which tends to justify our value for the effective number of molecules. A detailed analysis of our fitting is presented in Fig. 5. The deviation $\sigma$ is defined as the sum of the square of the differences between the theoretical and experimental current values at every bias. The smallest value of the deviation, as a function of $E_{f}-E_{\mathrm{HOMO}}$ and the effective number of well-contacted molecules, represents the best fit in the $I-V$. We observe a clear minimum for $\sigma$ in Fig. 5 for the corresponding value of $E_{f}-E_{\mathrm{HOMO}}=1.3 \mathrm{eV}$ and effective number of molecules $\sim 425$.

An analysis of conduction through the ODT molecule is presented in Fig. 6. It clearly shows that the conduction is taking place only in the tunneling regime for the entire range of the applied bias and transmission remains very low inside the $\mu_{1}-\mu_{2}$ window. Fig. 7 (a) shows the length dependence of the transmission coefficients for three different lengths of alkane dithiol molecules. As the experimental $I-V$ data for dodecane dithiol (C12) and hexadecane dithiol (C16) are not available at this time in the same nanopore setup, we are not able to pinpoint the position of the Fermi level for these two molecules independently. Instead, we have used the same $V_{c}$ value (1.55 eV from the ODT calculations) to generate all the three curves in Fig. 7(a). We observe that the transmission decreases exponentially with the increase in the molecu-
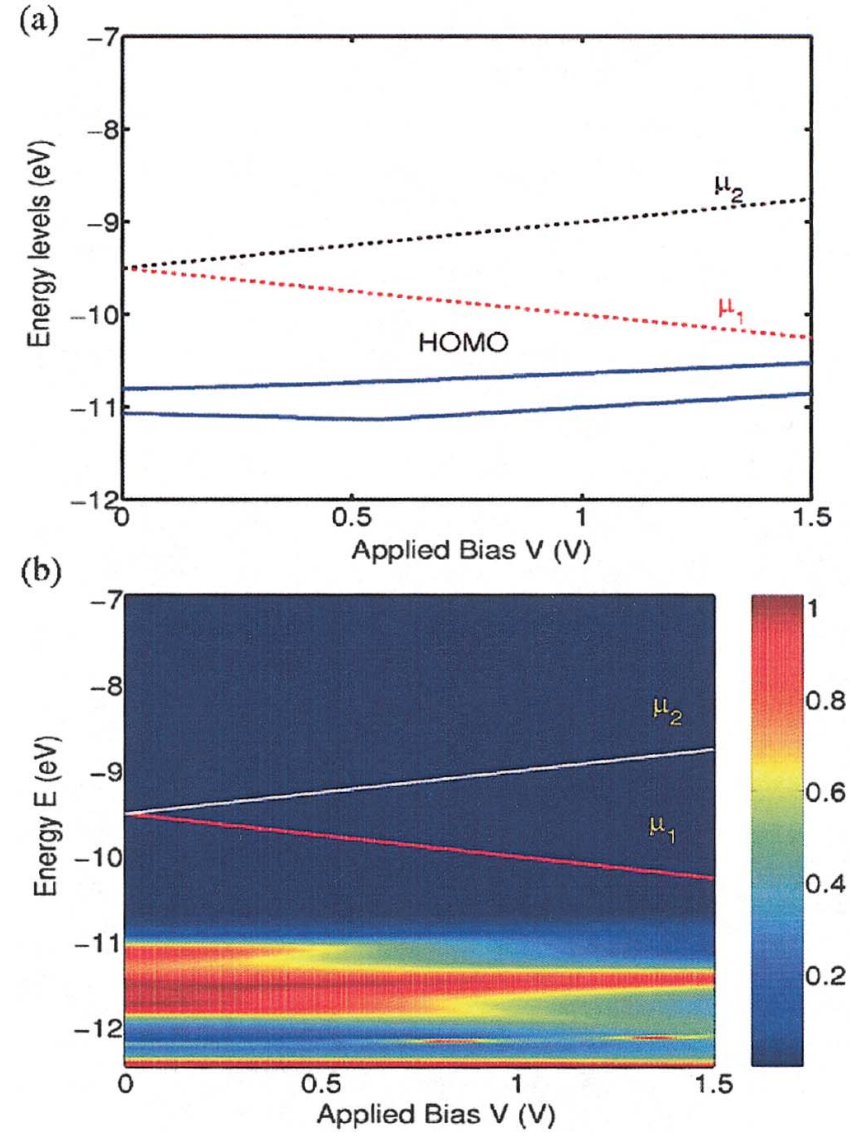

FIG. 6. (Color) (a) Energy level as a function of applied bias and (b) the corresponding transmission coefficients as a function of energy and applied bias in a color plot at $E_{f}-E_{\mathrm{HOMO}}=1.3 \mathrm{eV}$. It is evident that the conduction remains in the tunneling regime with low transmission coefficients in the entire bias range.
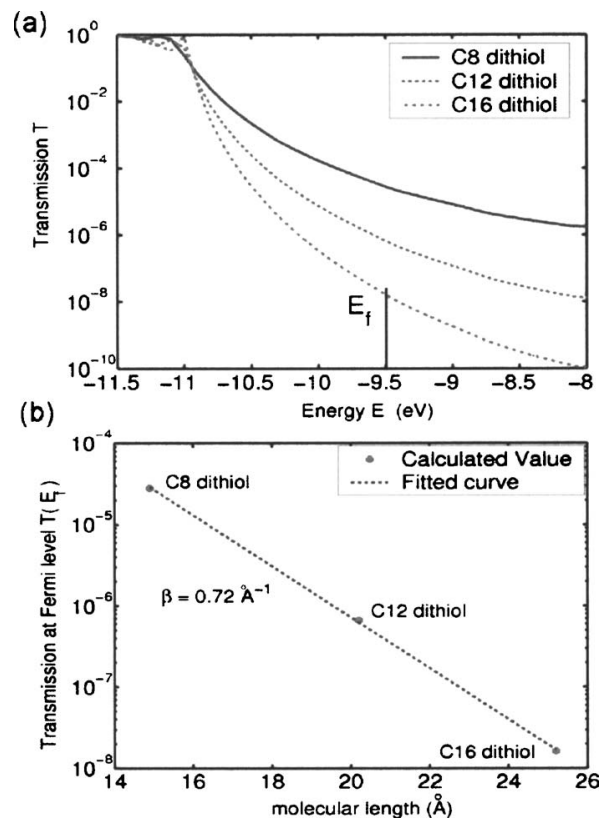

FIG. 7. (a) Equilibrium transmission coefficient as a function of energy for three alkane dithiol molecules of different length with the same $V_{c}$ value $(1.55 \mathrm{eV})$ for all three curves and (b) the curve fitting for the calculation of the decay coefficient $\beta$. Our calculated value of $\beta$ for alkane dithiol molecules, $0.72 \AA^{-1}$, is comparable to experimentally observed values. 
lar length as expected for coherent electron conduction. From the transmission we estimate the decay coefficient $\beta$ using the following equation:

$$
T\left(E_{f}\right)=T_{0}\left(E_{f}\right) \exp (-\beta d) .
$$

Figure 7(b) shows the fitted curve from which the $\beta$ value is extracted. The decay coefficient for alkanethiol molecules obtained from many different experimental setups and techniques have been previously reported; for example, Wang et al. reported a $\beta$ value between 0.83 and $0.72 \AA^{-1}$ using a nanopore setup, ${ }^{40}$ Holmlin et al. reported a $\beta$ value of $0.87 \AA^{-1}$ by mercury-drop experiments ${ }^{53}$ and Cui et al. reported a $\beta$ value of $0.79 \AA^{-1}$ for many alkanethiol molecules by using a conducting atomic force microscope technique. ${ }^{54}$ We see that our value of $\beta=0.72 \AA^{-1}$ is comparable to the experimental results.

A theoretical treatment of the same experimental $I-V$ characteristics studied here has been reported previously by Wang et $a l .^{40}$ In that treatment, the fitting for a given value of area $A$ of the nanopore and the molecular length $d$ (length of the barrier) is performed using a modified Simmons model $^{53,55}$ with two fitting parameters: the height of the 1-D potential barrier $\phi_{0}$ and a nonideality factor $\alpha$ corresponding to a nonrectangular-shaped barrier or the effective mass of the electron. They observed an excellent match with the experimental $I-V$ for the particular values of $\phi_{0}=1.2 \mathrm{eV}$ and $\alpha=0.59$. The 1 -D barrier height $\phi_{0}=1.2$ obtained by the authors using their simple model corresponds well with our value of $E_{f}-E_{\mathrm{HOMO}}=1.3 \mathrm{eV}$ calculated with a fully atomistic model for the molecule and the contacts with rigorous 3-D self-consistent electrostatics. It is difficult to connect the parameter $\alpha$ with any of our parameters in a straightforward manner. This parameter $\alpha$ depends in a complicated way on the self-consistent potential, the couplings among molecular orbitals, and the molecule-gold coupling, none of which is a fitting parameter in our calculations. If the transport was dominated by a single orbital, it may be possible to extract an effective mass from the hopping terms, but this is not the case for the nonconjugated molecules considered here. We believe that the extended Hückel coupling matrices within the molecule describe, in effect, the effective mass alluded to in the calculations by the Reed group. Our other fitting parameter, the effective number of well-contacted molecules, can be loosely connected to the area $A$ of the nanopore.

\section{SINGLE MOLECULE TRANSISTOR: GATE EFFECTS ON $\boldsymbol{l} \boldsymbol{V}$}

In this section we discuss gate effects on the molecular $I-V$ and present a few results for three terminal systems obtained using Huckel $I-V$ 3.0. We show that in addition to the electrostatic effects due to the gate electrodes, electronic properties of individual molecules play an important role in molecular transistors. Unfortunately, there exists almost no experimental data in the literature that shows any significant gate-induced effects on molecular $I-V$. Hence, the results we present in this section are made without connecting to any experimental results. Our objective is to showcase one of the major strengths of our model, i.e., the description of the potential profile inside the molecule even in the presence of an

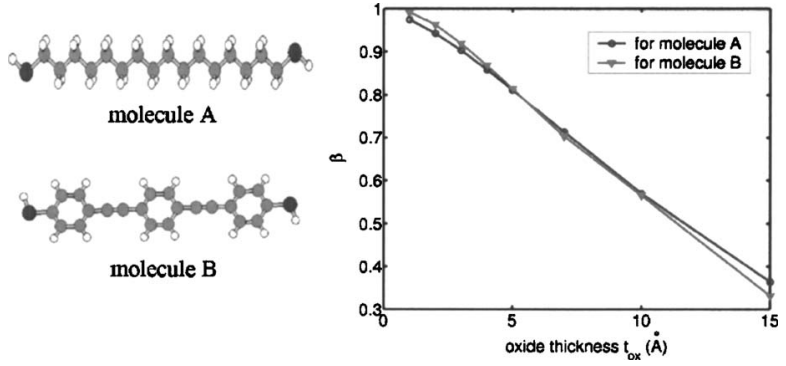

FIG. 8. Estimate of gate control $\beta$ for two molecules (shown on the left) as a function of oxide thickness with four gate plates around the molecule and with oxide dielectric constant $\epsilon_{\mathrm{ox}}=3.9$. The value of $\beta$ is averaged over the molecular length. As both molecules are roughly of the same length $(\sim 20 \AA)$ gate control for both of them is almost the same. To obtain good gate control $(\beta \sim 1)$ the gate electrodes need to be placed prohibitively close to the molecules $(\sim 2 \AA)$.

additional terminal as a gate electrode. In addition, we elucidate the role of electronic properties like metal-inducedgap states (MIGS) or HOMO-LUMO gap on transistor action. We illustrate these points by comparing the transistor performance of two molecules of similar lengths but of very different electronic structures.

Figure 8 shows the two different molecular structures that will be studied in this section. Molecule $A$ is a nonplanar alkanethiol chain (hexadecane dithiol) which is insulating in nature with a large HOMO-LUMO gap $(\sim 8-10 \mathrm{eV})$. Molecule $B$ is a benzene-based conjugated molecule [oligo(phenylene ethynylene) OPE] with thiol groups at both ends. The HOMO-LUMO gap of OPE molecule is much smaller $(\sim 2-4 \mathrm{eV})$. Both molecules are connected by sulphur end atoms to the gold source and drain electrodes. In Huckel $I-V 3.0$ we can add as many as four rectangular gate electrodes around the molecule, two in plane and two out of plane. An oxide layer is placed in between the molecule and the gate plates as an insulating medium (see Fig. 1). Since the main effect of the oxide is electrostatic insulation, with no consideration for gate leakage effects in this treatment, we adopt a continuum model for the oxide, specified simply in terms of its thickness $t_{\mathrm{ox}}$ and its dielectric constant $\epsilon_{\mathrm{ox}}$.

The electrostatic gate control of the molecular device depends on the oxide thickness, the oxide dielectric constant, and the length of the molecule. We have chosen molecules $A$ and $B$ as such that they are of similar length $(\sim 20 \AA)$ so that the differences in their transistor characteristics arise exclusively from their electronic, as opposed to their electrostatic properties. A parameter $\beta$ can be defined as a measure of the gate control as follows: ${ }^{56}$

$$
\beta=\frac{\partial U_{L}}{\partial V_{G}},
$$

where $U_{L}$ is the Laplace potential inside the molecule and $V_{G}$ is the applied gate bias. The parameter $\beta$ can take any value between 0 and 1 and the maximum gate control is obtained when $\beta=1$. We see from Fig. 8 that $\beta$ values for both molecules $A$ and $B$ are roughly same. We also notice that the gate control is considerably low $(\beta \sim 0.5)$ when the gate electrodes are placed at a reasonable distance $\left(t_{\mathrm{ox}} \sim 10 \AA\right)$ from the molecule. This point is further emphasized in Fig. 9. 

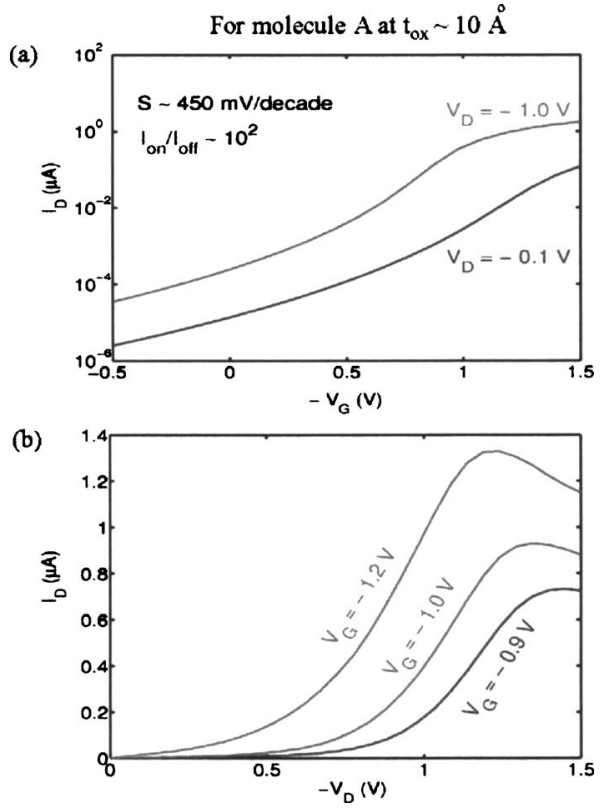

FIG. 9. Transistor characteristics $\left(I_{D^{-}} V_{G}\right.$ and $I_{D^{-}} V_{D}$ curves) for molecule $A$ at oxide thickness $t_{\mathrm{ox}} \sim 10 \AA$ with a number of gate electrodes $=4$ and oxide dielectric constant $\epsilon_{\mathrm{ox}}=3.9$. The values of subthreshold swing $S$ $(\sim 450 \mathrm{mV} /$ decade $)$ and $I_{\text {on }} / I_{\text {off }}$ ratio $\left(\sim 10^{2}\right)$ are poor. The $I_{D}-V_{D}$ curves show no saturation of current. Overall, molecule $A$ shows very poor transistor performance operating at $t_{\mathrm{ox}} \sim 10 \AA$.

From Fig. 9(a) we observe that the subthreshold swing $(\sim 450 \mathrm{mV} /$ decade) of molecule $A$ is too high when operating at $t_{\mathrm{ox}} \sim 10 \AA$ and there is almost no gate modulation. Besides, the $I_{D}-V_{D}$ curves [Fig. 9(b)] do not show any current saturation. It is evident from Figs. 8 and 9 that for a molecule of a length of around $20 \AA$, good gate control (thus good transistor performance) can be achieved only when the oxide thickness is much smaller than $10 \AA$.

For the comparison of transistor characteristics between molecule $A$ and molecule $B$ we choose an oxide thickness of $t_{\mathrm{ox}} \sim 2 \AA$. We understand that an oxide layer of only $2 \AA$ thickness may be too thin to be reliable as an insulator. Besides, it becomes difficult to justify treating the oxide layer as a continuum at this thickness. However, we observe the

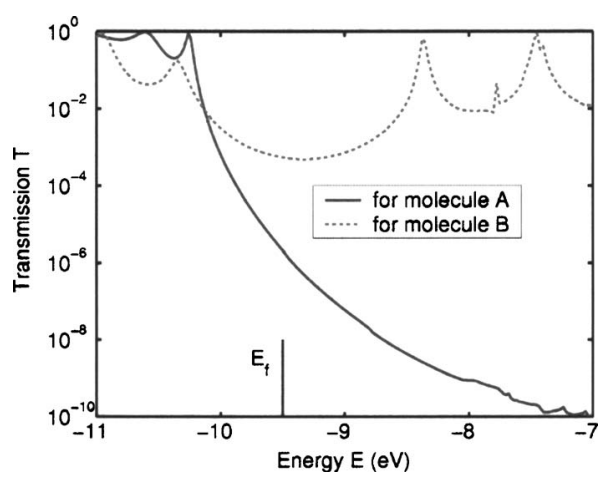

FIG. 11. A comparison of the equilibrium transmission coefficients as a function of energy between molecular systems $A$ and $B$. Transmission coefficients for molecule $A$ are much lower around $E_{f}$ due to fewer MIGS. This is the main reason for the better performance of molecule $A$ as a switching device compared to that of molecule $B$. Besides, the HOMO-LUMO gap of molecule $A$ is much larger than that of molecule $B$ which also affects the transistor action.

best transistor performance for both molecules at around $t_{\mathrm{ox}}=2 \AA$, which helps to identify more clearly the differences in the transistor performance between the two molecular transistors, $A$ and $B$.

The transistor characteristics presented in Fig. 10 clearly show that molecule $A$ acts as a better switching device compared to molecule $B$. Transistor $A$ can be turned on or off much more easily as its subthreshold swing $(\sim 150 \mathrm{mV} /$ decade $)$, defined as $S=\left(d\left(\log I_{D}\right) / d V_{G}\right)^{-1},{ }^{57}$ is much lower than that of transistor $\mathrm{B}(\sim 350 \mathrm{mV} /$ decade $)$. In addition, the on and the off states are more clearly defined for transistor $A$ as its $I_{\text {on }} / I_{\text {off }}$ ratio $\left(\sim 10^{4}\right)$ is much higher than that $\left(\sim 10^{2}\right)$ of transistor $B$. The reasons for these obvious differences in the transistor performance of these two molecules can be understood from the plot of equilibrium transmission coefficient (Fig. 11). The figure shows that the transmission is much lower in the vicinity of $E_{f}$ for molecule $A$ compared to molecule $B$ due to the lack of metal-inducedgap states (MIGS). However, the transmissions around the HOMO level for both molecules are roughly the same. As transmission is proportional to the current it can be assumed
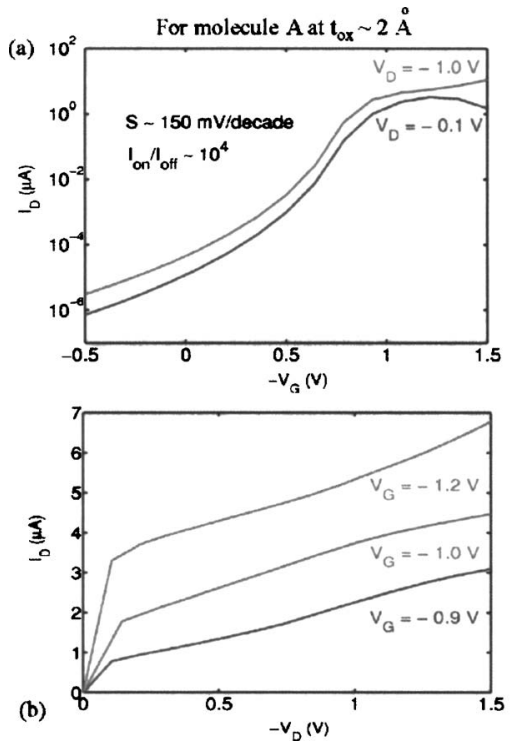
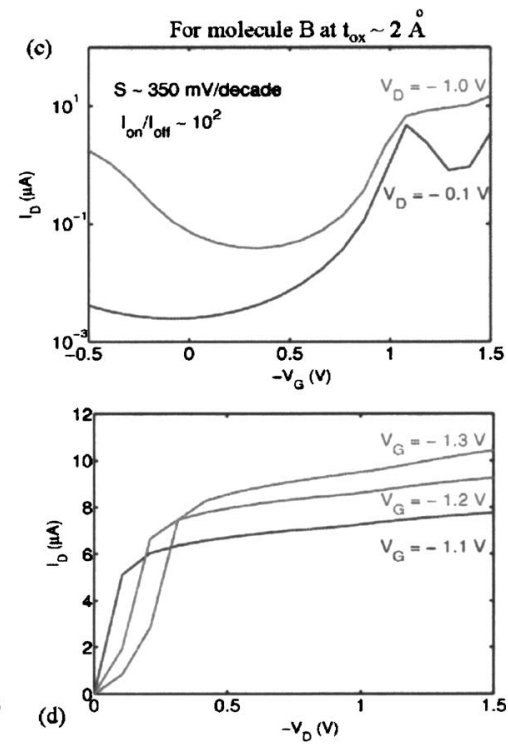

FIG. 10. Transistor characteristics for molecule $A$ [(a) and (b)] and molecule $B[(\mathrm{c})$ and (d)] at oxide thickness $t_{\mathrm{ox}} \sim 2 \AA$ with a number of gate electrodes $=4$ and oxide dielectric constant $\epsilon_{\mathrm{ox}}=3.9$. The value of $E_{f}-E_{\mathrm{HOMO}}$ is set to be 0.7 and $0.9 \mathrm{eV}$ for molecules $A$ and $B$, respectively. The main difference in transistor performance between these two molecules is in the values of subthreshold swing $S$ and $I_{\text {on }} / I_{\text {off }}$ ratio. For molecule $A$ we obtain $S \sim 150 \mathrm{mV} /$ decade and $I_{\text {on }} / I_{\text {off }} \sim 10^{4}$ which are much better for switching action compared to those of molecule $B\left(S \sim 350 \mathrm{mV} /\right.$ decade and $\left.I_{\text {on }} / I_{\text {off }} \sim 10^{2}\right)$. For both molecules the subthreshold swing is obtained from the $I_{D}-V_{G}$ curve at $V_{D}=-0.1 \mathrm{~V}$ in the linear region between $V_{G}=-0.5 \mathrm{~V}$ and $V_{G}=-0.7 \mathrm{~V}$. Both molecules show relatively low current saturation [(b) and (d)] whereas, irregular behavior (current is going down with higher $V_{G}$ ) is observed at low $V_{D}$ for molecule $B$. Molecule $B$ also shows bipolar transistor behavior (c) as current increases in both polarities of $V_{G}$. 
For molecule A
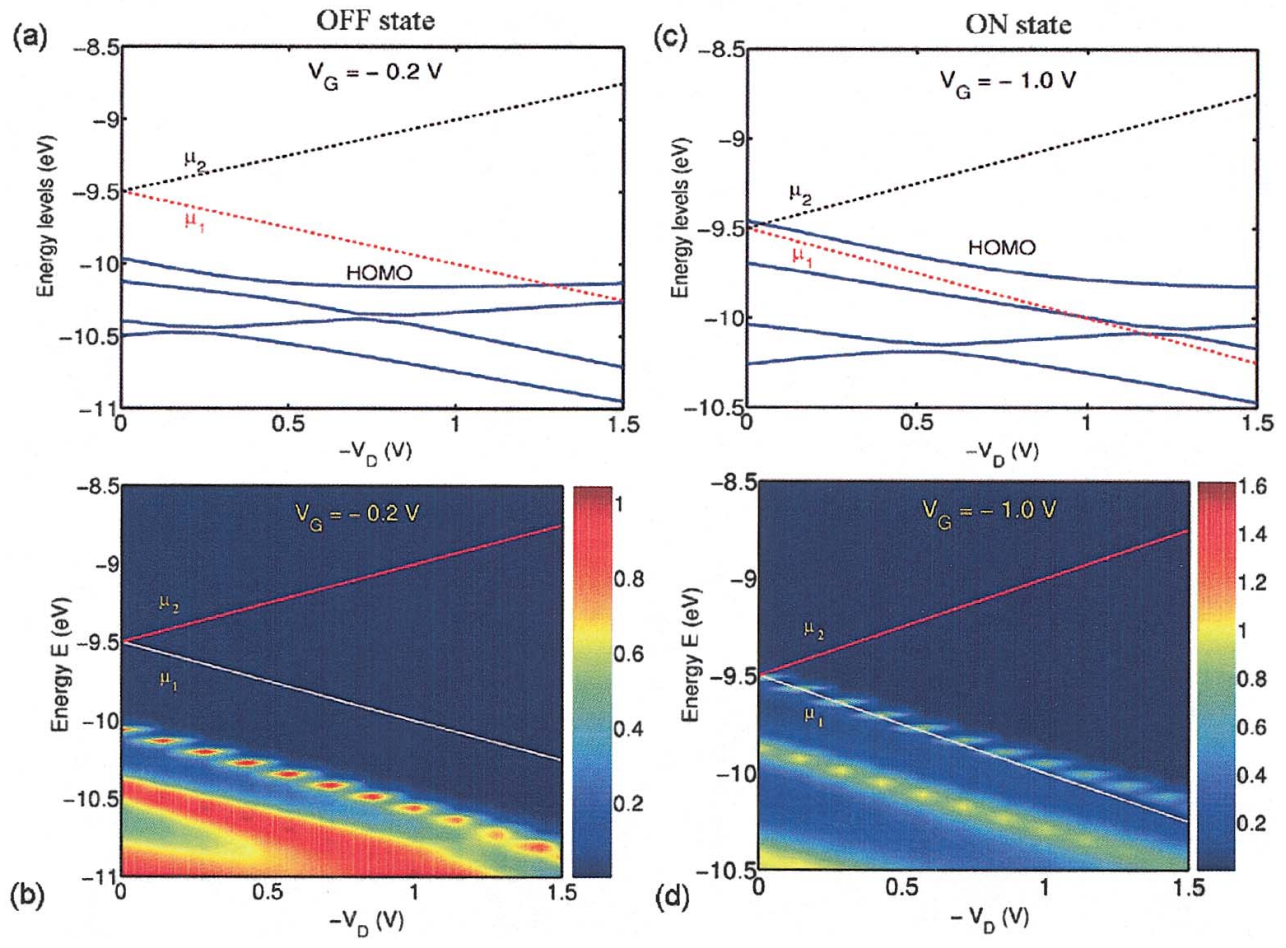

FIG. 12. (Color) Energy level as a function of drain bias and the corresponding color plot of the transmission as a function of energy and drain bias for molecule $A$ at $t_{\mathrm{ox}} \sim 2 \AA$ in off [(a) and (b)] and on [(c) and (d)] states. The source and drain electrochemical potentials are shown by $\mu_{1}$ and $\mu_{2}$, respectively. At small gate bias $[(a)$ and (b)] the current remains very low as tunneling conduction takes place through the HOMOLUMO gap. The current level increases with high gate bias [(c) and (d)] as the HOMO level comes inside the $\mu_{1}-\mu_{2}$ window. However, the current then starts to saturate with increasing drain bias as no new energy levels come inside the $\mu_{1}-\mu_{2}$ window. that $I_{\text {on }} / I_{\text {off }}$ is equivalent to $T_{\text {on }} / T_{\text {off }}$, where $T_{\text {off }}$ is the transmission around $E_{f}$ and $T_{\text {on }}$ is the transmission around the HOMO level. Thus it becomes evident why the $I_{\text {on }} / I_{\text {off }}$ ratio for molecule $A$ is much higher than that of molecule $B$. Similarly, the subthreshold slope is related to the rate of change of transmission inside the $E_{f}$ to $E_{\text {HOMO }}$ energy range. The change in transmission is much steeper for molecule $A$ (Fig. 11) and subsequently, its subthreshold slope is much higher than that of molecule $B$.

When a bias is applied to the gate electrodes it raises or lowers the molecular energy levels according to the bias polarity. With source electrode grounded, the Laplace potential $U_{L}$ inside the molecule can be written as:

$$
U_{L}=\beta\left(-q V_{G}\right)+\frac{(1-\beta)}{2}\left(-q V_{D}\right),
$$

where $\beta$ is the gate control parameter defined earlier. If the gate control is ideal, i.e., $\beta=1$, then the Laplace potential becomes $U_{L}=-q V_{G}$ which essentially holds the gate electrodes at a fixed potential with respect to the source. ${ }^{56}$ This means the molecular energy levels will follow the source chemical potential under the applied drain bias as long as the Laplace potential remains the dominant factor. We observe from Fig. 12 that the molecular energy levels are, indeed, following the source chemical potential $\mu_{1}$.

It is evident from Figs. 12(a) and 12(b) that at low gate bias the conduction takes place inside the HOMO-LUMO gap (tunneling regime) which keeps the system in the off state. Under large gate bias the molecular levels move up sufficiently enough that the system enters the resonant tunneling regime where conduction takes place through the HOMO level [Figs. 12(c) and 12(d)] which in turn makes the current level high and switches the system to the on state. This is the basic switching mechanism for the molecular transistor. The current saturation at high drain bias in the on state can be explained as follows: for a fixed gate bias in the on state [Figs. 12(c) and 12(d)] when the drain bias is increased, the molecular energy levels remain fixed with respect to $\mu_{1}$ under strong gate control conditions $(\beta \sim 1)$ and at some point the current becomes almost saturated as no new level crosses $\mu_{2}$ and the transmission inside the $\mu_{1}$ $-\mu_{2}$ window remains very low. As the density of states inside the HOMO-LUMO gap remains small but nonzero for both of the molecules, the current keeps on increasing steadily without attaining complete saturation [Figs. 10(b) and $10(\mathrm{~d})$ ]. It is worth pointing out that realizing a transistor like molecule $A$ showing impressive subthreshold swing may require additional considerations of threshold voltage adjustment, power supply, etc. which have not been discussed here.

\section{CONCLUSION}

In this paper we presented our model Huckel $I-V 3.0$ which provides a practical simulator for quantum transport in molecules including a semiempirical description of the full 3-D electrostatics and employs just the location of the Fermi energy as a single adjustable parameter. We applied our model for quantitative analysis of experimentally observed $I-V$ characteristics of alkane dithiol molecules. Our calculations showed good agreement with the experiments. We also presented a comparative assessment on molecular transistor action. Currently, we are working on improving our model by adding a proper atomistic description of tunneling through vacuum in scanning tunneling microscope (STM) measurements. We are also looking into the prospect of incorporating silicon contacts in our model to complement our first-principles treatments of these hybrid systems. ${ }^{35}$ 


\section{ACKNOWLEDGMENTS}

We would like to thank Takhee Lee, R. Reifenberger, and D. Janes for useful discussions. This work has been supported by the NSF Network for Computational Nanotechnology and the US Army Research Office (ARO) under Grant Nos. EEC-0228390 and DAAD19-99-1-0198.

${ }^{1}$ H. Ohnishi, Y. Kondo, and K. Takayanagi, Nature (London) 395, 780 (1998).

${ }^{2}$ M. A. Reed, C. Zhou, C. J. Muller, T. P. Burgin, and J. M. Tour, Science 278, 252 (1997).

${ }^{3}$ S. A. Getty, C. Engtrakul, L. Wang et al., cond-mat/0409433.

${ }^{4}$ G. E. Poirier and M. J. Tarlov, Langmuir 10, 2853 (1994); G. Leatherman, E. N. Durantini, D. Gust et al., J. Phys. Chem. B 103, 4006 (1999).

${ }^{5}$ B. Chen and J. M. Metzger, J. Phys. Chem. B 103, 4447 (1999).

${ }^{6}$ J. Chen, M. A. Reed, A. M. Rawlett, and J. M. Tour, Science 286, 1550 (1999)

${ }^{7}$ N. P. Guisinger, M. E. Greene, R. Basu, A. S. Baluch, and M. C. Hersam, Nano Lett. 4, 55 (2004).

${ }^{8}$ P. Avouris, P. G. Collins, and M. S. Arnold, Science 292, 706 (2001).

${ }^{9}$ A. Bachtold, P. Hadley, T. Nakanishi, and C. Dekker, Science 294, 1317 (2001).

${ }^{10}$ J.-O. Lee, G. Lientschnig, F. Weirtz et al., Nano Lett. 3, 113 (2003).

${ }^{11}$ J. Park, A. N. Pasupathy, J. I. Coldsmith et al., Nature 417, 722 (2002).

${ }^{12}$ L. H. Yu and D. Natelson, Nanotechnology 15, S517 (2004).

${ }^{13}$ J. R. Petta, S. K. Slater, and D. C. Ralph, Phys. Rev. Lett. 93, 136601 (2004).

${ }^{14}$ W. Wang, T. Lee, I. Kretzschmar, and M. A. Reed, Nano Lett. 4, 643 (2004).

15 J. G. Kushmerick, J. Lazorcik, C. H. Patterson, R. Shashidar, D. S. Seferos, and G. C. Bazan, Nano Lett. 4, 639 (2004).

${ }^{16}$ W. Ho, X. H. Qiu, and G. V. Nazin, Phys. Rev. Lett. 92, 206102 (2004).

${ }^{17}$ Y. Cui, Q. Wei, H. Park, and C. M. Lieber, Science 293, 1289 (2001).

${ }^{18}$ R. P. Andres, T. Bein, M. Dorogi et al., Science 272, 1323 (1996).

${ }^{19}$ J. Reichert, R. Ochs, D. Beckmann, H. B. Weber, M. Mayor, and H. V. Löhneysen, Phys. Rev. Lett. 88, 176804 (2002).

${ }^{20}$ C. Kergueris, J.-P. Bourgois, S. Palacin, D. Esteve, C. Urbina, M. Magoga, and C. Joachim, Phys. Rev. B 59, 12505 (1999).

${ }^{21}$ C. Joachim and J. K. Gimzewski, Chem. Phys. Lett. 265, 353 (1997).

${ }^{22}$ W. Tian, S. Datta, S. Hong, R. Reifenberger, J. I. Henderson, and C. P. Kubiak, J. Chem. Phys. 109, 2874 (1998).

${ }^{23}$ S. Hong, R. Reifenberger, W. Tian, S. Datta, J. Henderson, and C. P. Kubiak, Superlattices Microstruct. 28, 289 (2000).

${ }^{24}$ M. Magoga and C. Joachim, Phys. Rev. B 59, 16011 (1999).

${ }^{25}$ E. G. Emberly and G. Kirczenow, Phys. Rev. B 62, 10451 (2000).

${ }^{26}$ L. E. Hall, J. R. Reimers, N. S. Hush, and K. Silverbrook, J. Chem. Phys. 112, 1510 (2000)

${ }^{27}$ M. Paulsson and S. Stafström, Phys. Rev. B 64, 035416 (2001).

${ }^{28}$ M. Di Ventra, S. T. Pantelides, and N. D. Lang, Phys. Rev. Lett. 84, 979 (2000)

${ }^{29}$ P. S. Damle, A. W. Ghosh, and S. Datta, Phys. Rev. B 64, 201403 (2001).

${ }^{30}$ J. J. Palacios, A. J. Perez-Jimenez, E. Lous, and J. A. Verges, Phys. Rev. B 64, 115411 (2001).

${ }^{31}$ J. Taylor, H. Gou, and J. Wang, Phys. Rev. B 63, 245407 (2001).
${ }^{32}$ Y. Q. Xue, S. Datta, and M. A. Ratner, J. Chem. Phys. 115, 4292 (2001).

${ }^{33}$ K. Stokbro, J. Taylor, M. Brandbyge, J.-L. Mozos, and P. Ordejon, Comput. Mater. Sci. 27, 151 (2003).

${ }^{34}$ J. M. Seminario, A. G. Zacarias, and J. M. Tour, J. Phys. Chem. 103, 7883 (1999)

${ }^{35}$ T. Rakshit, G.-C. Liang, A. W. Ghosh, and S. Datta, Nano Lett. 4, 1803 (2004).

${ }^{36}$ F. Zahid, M. Paulsson, and S. Datta, in Advanced Semiconductor and Organic Nano-Techniques, edited by H. Morkoc (Academic, New York, 2003), Vol. 3.

${ }^{37}$ F. Zahid, A. W. Ghosh, M. Paulsson, E. Polizzi, and S. Datta, Phys. Rev. B 70, 245317 (2004).

${ }^{38}$ G. C. Liang, A. W. Ghosh, M. Paulsson, and S. Datta, Phys. Rev. B 69, 115302 (2004).

${ }^{39}$ The source codes of Huckel $I-V 3.0$ will be made available through Purdue Nanohub in near future.

${ }^{40}$ W. Wang, T. Lee, and M. A. Reed, J. Phys. Chem. B 108(48), 18398 (2004).

${ }^{41}$ J. Howell, A. Rossi, D. Wallace, K. Haraki, and R. Hoffman, FORTICON8, QCPE Program 545, Department of Chemistry, Cornell University, Ithaca, NY, 14853

${ }^{42}$ S. Datta, Electronic Transport in Mesoscopic Systems (Cambridge University Press, Cambridge, 1995).

${ }^{43}$ R. Zeller, J. Deutz, and P. H. Dederichs, Solid State Chem. 44, 993 (1982).

${ }^{44}$ In the nonequilibrium situation, contour integration is employed only up to one contact electrochemical potential (either $\mu_{1}$ or $\mu_{2}$, depending on the bias polarity). Inside the $\mu_{1}-\mu_{2}$ window, the integration is performed using a real energy grid.

${ }^{45}$ S. Datta, W. Tian, S. Hong, R. Reifenberger, J. I. Henderson, and C. P. Kubiak, Phys. Rev. Lett. 79, 2530 (1997).

${ }^{46}$ The results presented in this paper use only the molecular system as the device region. no part of the gold contacts are included in the selfconsistent calculations. Although our model takes into account the effects of image charges due to the contacts, it would be useful to include a few layers of the contacts in the calculations for a better description of the gold-sulphur bond. However, due to practical difficulties, e.g., determining reliable CNDO parameters for gold, it is left for future research.

${ }^{47}$ E. Polizzi and N. Ben Abdallah, Phys. Rev. B 66, 245301 (2002).

${ }^{48}$ J. A. Pople and G. A. Segal, J. Chem. Phys. 44, 3289 (1966).

${ }^{49}$ J. N. Murell and A. J. Harget, Semi-empirical SCF Theory of Molecules (Wiley, New York, 1972).

${ }^{50}$ H. Sellers, A. Ulman, Y. Shnidman, and J. E. Eilers, J. Am. Chem. Soc. 115, 9389 (1993).

${ }^{51}$ M. J. Frisch, G. W. Trucks, H. B. Schlegel et al., GAUsSIAN 98, Revision a.7, Gaussian Inc., Pittsburgh PA, 1998.

${ }^{52}$ B. Xu and N. J. Tao, Science 301, 1221 (2003).

${ }^{53}$ R. Holmlin, R. Haag, M. L. Chabinyc, R. F. Ismagilov, A. E. Cohen, A. Terfort, M. A. Rampi, and G. M. Whitesides, J. Am. Chem. Soc. 123, $5075(2001)$

${ }^{54}$ X. D. Cui, X. Zarate, J. Tomfohr et al., Nanotechnology 13, 5 (2002).

${ }^{55}$ G. Simmons, J. Appl. Phys. 34, 1793 (1963).

${ }^{56} \mathrm{P}$. Damle, T. Rakshit, M. Paulsson, and S. Datta, IEEE Trans. Nanotechnol. 1, 145 (2002).

${ }^{57}$ Y. Taur and T. H. Ning, Fundamentals of Modern VLSI Devices (Cambridge University Press, Cambridge, 1998) 